\title{
(Research Article) \\ Integration of BCI and Eye Gaze Tracker to Control Mouse
}

\author{
H. M. Mehta ${ }^{1 *}$, Prof. M. P. Patel ${ }^{2}$, Dr. C. H. Vithalani ${ }^{3}$ \\ ${ }^{1 * 2}$ Department of Electronics \& Communication Engineering, B. H. Gardi College of Engineering and Technology, Rajkot, Gujarat, INDIA \\ ${ }^{3}$ Department of Electronics \& Communication Engineering, Government Engineering College, Rajkot, Gujarat, INDIA
}

\begin{abstract}
This paper represents how EEG based BCI module can be used with HCI devices for the physically disabled people to interact with the surrounding world. EEG is one of the imaging technology which records electrical activity spontaneously generated from the brain. Owing to the less accuracy of this cheaper and affordable BCI module, it cannot be used where many control signals are required to operate any device. In this paper, Neurosky's Brainwave starter kit has been used as a BCI module which has a mono channel sensor to acquire the brain signals. To increase the ability of such BCI module, Eye Gaze Tracker (Human Computer Interface) has been attached with it. By merging these two techniques, windows software of mouse controller is developed using Python language and OpenCV as a third party module. This python program file can also be used in other operating systems such as Linux, OSX etc. This software can be extremely useful to ALS (Amyotrophic Lateral Sclerosis) patients, paralyzed people, who are lacking any kind of muscular movement to control any devices.
\end{abstract}

Keywords: BCI (Brain Computer Interface), EEG (Electroencephalography), HCI (Human Computer Interface), Eye Gaze Tracker, Python, OpenCV.

\section{Introduction}

In India, there are about 5 million physically disabled people who are suffering from various diseases or some are disabled by born. They are unable to communicate with the surrounding world so effectively that they often come under depression. So requirement of making devices for them has been emerged and various types of BCI devices have been invented for their comfort and there still a lot of research is remaining to be done to use it effectively and which should be affordable to everyone.

The BCI devices are used to acquire the brain signals and to control any devices using them. When a person thinks about something, neurons get fired in his mind and accordingly patterns of these neural activities are generated and that is in the form of electrical signals. So it can be measured by electrodes either placed on the scalp or on the surface of cortex or inside the skull. Based on this, [1] classifies BCI devices are into three categories: 1) Invasive method, 2) Partially Invasive method, 3) Noninvasive method. Invasive devices are implanted inside the skull by a critical surgery in which a chip is put inside the cortex which consists of hundreds of electrodes to measure the brain

${ }^{*}$ Corresponding Author: e-mail: hardikmmehta3@gmail.com ISSN 2320-7590

(C) 2018 Darshan Institute of Engg. \& Tech., All rights reserved signals. It can give very accurate result but due to surgery, scar tissue is formed which can degrade the signal quality. So this method causes pain to patients, implementation is costly and maintenance is also required. In Partially Invasive method, electrodes are implanted inside the skull but rest remains outside the brain. So the signal becomes weaker in comparison with the Invasive devices but less risk of forming scar tissue. [2] Shows that Non-invasive device measures the brain signals by putting electrodes over the scalp. There is no surgery required in this method of BCI. Though skull is about $7 \mathrm{~mm}$ thick, distortion occurs in the brain signals and it degrades the signal quality. But for the few controlling operations, it is quite good to use and affordable. So there should be some research towards increasing the accuracy of Noninvasive BCI.

In this work, EEG based Neurosky's brainwave starter kit is used which is a noninvasive BCI. There are many applications of this device mentioned below. Wireless mind controlled robot is made using this module in [3] which acquires the EEG signals using single channel sensor placed on the forehead and the signal feature is extracted using the Discrete Wavelet Transform so that blink strength detection can be made very accurate and by using it, wireless robot can be controlled. Another application is depicted in [4] which demonstrate the vigilance level of the driver. Drowsiness is the major problem scene in the drivers who must stay alert for a long period of time while driving. So BCI causes 
vibrations in the steering wheel to alert the driver and at the danger level, it alerts to his relatives/friends. A robotic mind controlled has been made by [5] and operated using the attention level and blink strength value which is provided by ThinkGear Connector built in the headset but since it has less accuracy, the robotic hand did not work properly. Various electrical devices can be tuned $\mathrm{ON}$ and $\mathrm{OFF}$ using $\mathrm{BCI}$ is shown in [6]. A web browser is also made based on the interest level of the user mentioned in [7]. When user reads about something and gets interested in it at that time he spends more time in that particular topic and while reading that page, his attention level also goes above the threshold defined by the programmer. The page with higher threshold value and spent more time is considered as his interest and then every time he gets similar pages on the start page as suggestions.

Due to less accuracy, some researchers have been trying to increase its performance by attaching various HCI devices sip/puff switch, speech recognition, gyroscope, eye gaze tracker with it [8] and just compared all the results. Some tried to move mouse cursor with gyroscope and mouse click using attention values [9] but ALS patients, paralyzed people are not able to move their head. So in this work, Eye Gaze Tracker has been attached with BCI to enhance its resolution. Viola and Jones developed cascade files and provided a great contribution in Image processing [10]. Fabian Timm developed an algorithm to track an Eye Gaze [11]. In this work, a python based software is made for controlling mouse which can be helpful to the patients to operate computer and interact with the surrounding world.

\section{Working of BCI}

Neurosky's MindWave Mobile works on Noninvasive method and has a single channel sensor to acquire the brain signals.

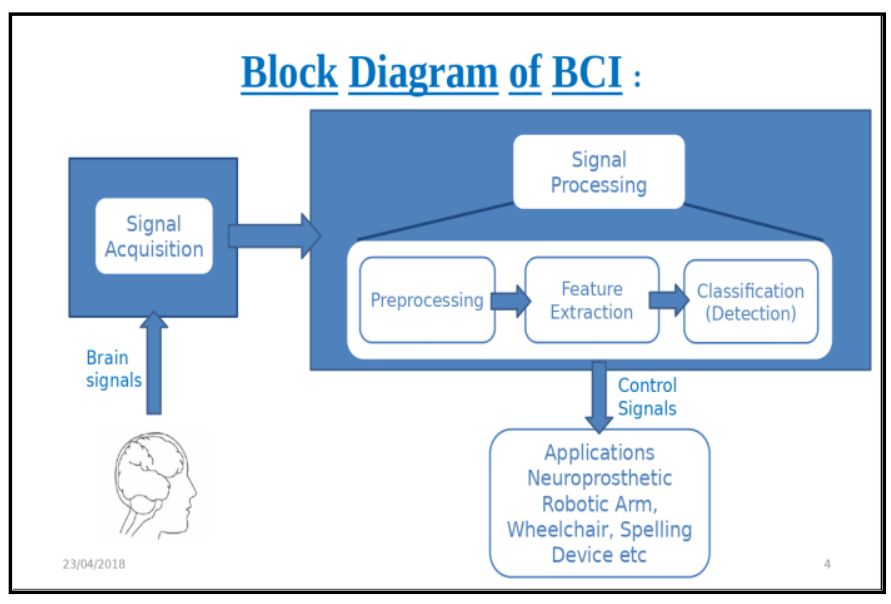

Figure 1. Block Diagram of BCI

Block Diagram of BCI is shown in Fig.1 which demonstrates the whole process. In the signal acquisition block, brain signals are acquired by the sensor. After that these signals are processed further in which noise is removed and signals are amplified in the preprocessing block. Thereafter the feature extraction components generate the discriminative characteristics for the improved signal which in turn results into the decrement in the size of the data applied to the classifiers. Classifiers (Classification components) translate the produced features into the device commands. Basically depending on a person's thinking capability and different situations and different time in which he thinks signals of varying frequencies are generated. Based on the frequencies, they are categorized into Delta $(0-4 \mathrm{~Hz})$, Theta $(4-8 \mathrm{~Hz})$, Alpha $(8-13 \mathrm{~Hz})$, Beta $(13-30 \mathrm{~Hz})$, Gamma $(30-100 \mathrm{~Hz})$ waves. All the signals are of low frequencies and in microvolts. Hence they can be easily contaminated and there will be distortion in them. Further a ThinkGear connector runs on the computer as a background process and is responsible for directing headset data from serial port to an open socket network. This headset is connected with the computer via Bluetooth. So, wireless connection provides a great mobility, too. There is also an eSense meter for converting raw signal value into Attention level and Meditation level (0 t o100). Attention level is high when a person concentrates at some thought or stares at something constantly. Whereas Meditation level is high when a person feels relax or inhale-exhale deeply like meditation. This eSense meter also measures the blink strength and it is very accurate, too. So using these three parameters, any wireless device can be controlled. Different wired BCIs are also available to control the wired device.

\section{Algorithm}

In this work, Python has been used to make a program of Eye Gaze Tracker, to make a GUI and to read the Attention values from the headset. Basically this work is all about Digital Image and Video Processing. First of all the user is being monitored by the any webcam either it is an inbuilt webcam or external webcam or IP-webcam. After that this algorithm starts searching the face provided that user must be present in front of the webcam all the time. As we know that to do all the process, the incoming frames must be converted into Grey Scale from RGB.

Face Detection is mainly working in this algorithm by the template matching algorithm because it gives more accurate results than the region based tracking, feature point based tracking methods. Template based matching method compares the current incoming frame with the thousands of reference frames already stored in the memory. These templates have been made in the different conditions such as different light conditions, different poses of a person, different shapes of persons etc. So incoming frames are compared with all the templates and gives very good result. In python, there is an XML file already available in Github named HaarCascade file which is the library of thousands of such templates. 
During this continuous detection of the face, this algorithm starts searching for eye positions. It also works on the same principle as Face Detection has i.e. template matching method. When an eye is detected, a rectangle is drawn from the starting pixel $(x, y)$ to the ending pixel $(x+w, y+h)$ of the region covering the whole eye where $\mathrm{w}$ is the width and $\mathrm{h}$ is the height of that region. Then histogram of that region is equalized to improve the contrast. To remove the noise, threshold and morphological operations (close, erode and open) are performed.

Then the specific range of pixels ( 0 to 5 ) is defined as a function and blobs are detected using the contour finding function. Blob is the connected region of similar pixels. After that delete the rightmost blob to detect the left eye as there is a mirror effect seen in the webcams. Area of this contour (blob) is defined and center of this contour is calculated using the moment function. Center has $\mathrm{X}$ and $\mathrm{Y}$ coordinates varying with the movements of the left eye. Now these coordinates are directly assigned to the mouse coordinates by subtracting the coordinates from the image size to avoid the mirroring effect. Otherwise the mouse cursor would move in the opposite direction of the eye movement. This is how mouse cursor can be controlled.

Neurosky's headset comes with the eSense meter which gives the direct readings of Attention Values, Meditation Values and Blink Strength based on the classification of the different frequencies. This eSense algorithm directly connects with the ThinkGear Connector which runs as the background process when the headset is connected to the laptop via Bluetooth. With the help of ThinkGear connector, user can get all the above readings in the python shell monitor. These readings vary with the concentration levels, with the level of relaxed situation and with the eye blink intervals. In this algorithm, values of Attention levels are used only. So when the user concentrates on some point or stares at something constantly, he will get the higher Attention values. By putting a threshold, mouse clicks can be controlled.

Python has the tkinter library which is able to create such a GUI that a user wants. In this work, a simple GUI is made in which there are five button situated in a single row using the grid function of the python-tkinter and when a user performs click on a button, then it changes its color into green color. Whereas if the user clicks on the other button, the previous button will come into its original position i.e. 'Sunken' position. So this algorithm can be used in many applications by calling the pre-defined function when the button is pressed.

Flowchart of this algorithm is mentioned in Fig.2.

\section{Implementation and Result}

In this work, Python tkinter has been used to make a Graphic User Interface (GUI) and OpenCV as a Third party module in Python is used for Eye Gaze Tracking.

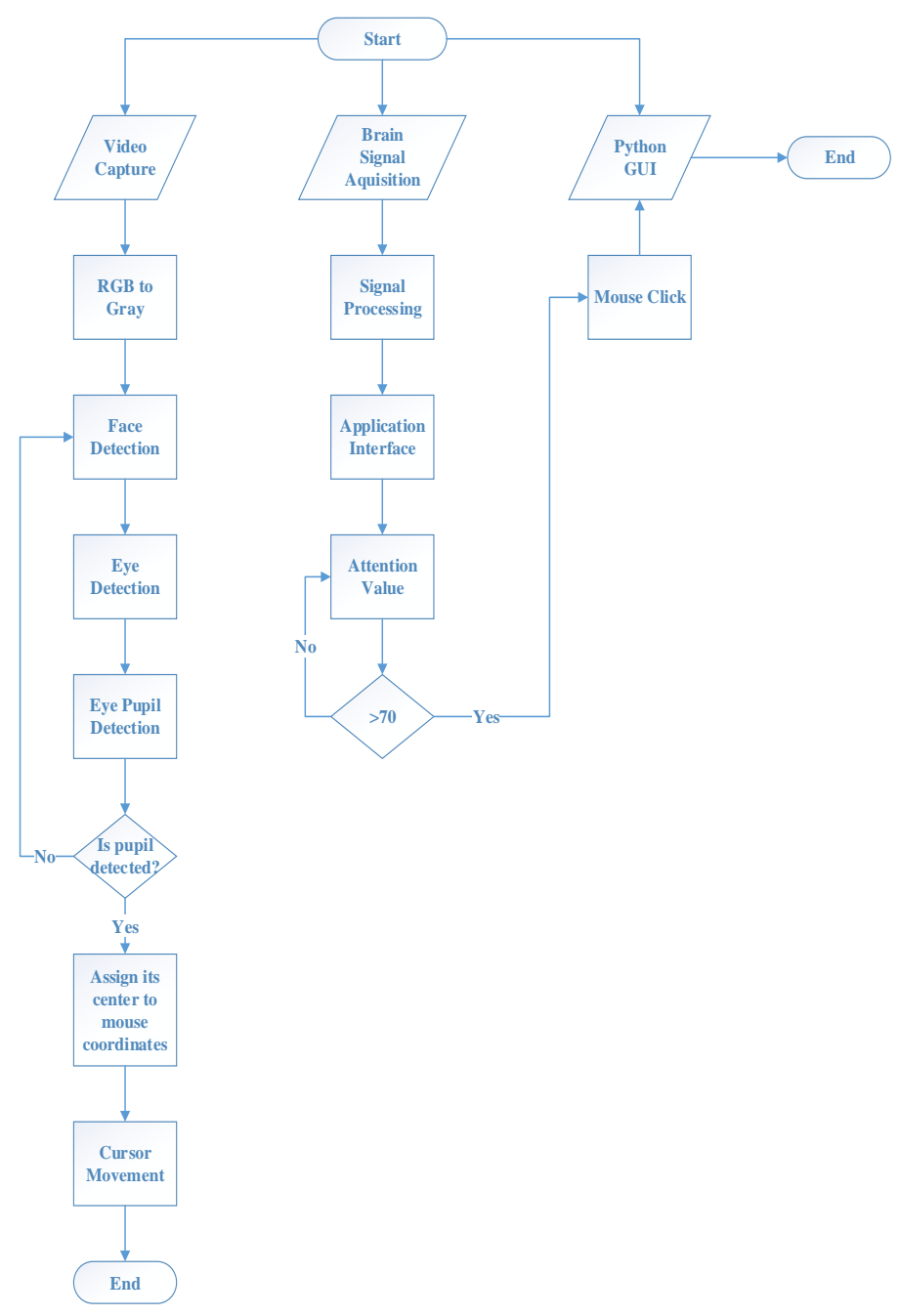

Figure 2. Flowchart of the entire architecture

In this algorithm, threshold value is set to 70 . So when the attention values go above the 70, algorithm will perform the clicking operation. Before this, user have to connect the headset with the laptop and enter the connected ' $\mathrm{COM}$ ' port into the program so that the algorithm gets all the values from the headset otherwise it will always show errors. The time required to click the mouse first time is calculated many times with the help of the python function time.clock () and shown in Table 1. Average of these different time responses is 14.4 seconds approximately which can be less in the other multichannel modules. The required time to make the first click done is different as it solely depends on the concentration level of the user. So the user needs to get trained for more accuracy. 
Table 1. The time required to click the mouse first time

\begin{tabular}{|c|c|}
\hline No. & Time Response (seconds) \\
\hline 1 & 19.5562423513 \\
\hline 2 & 7.1567866326 \\
\hline 3 & 28.1948711599 \\
\hline 4 & 9.4656892134 \\
\hline 5 & 13.7892359423 \\
\hline 6 & 25.6874156256 \\
\hline 7 & 5.1596357842 \\
\hline 8 & 7.3574159662 \\
\hline 9 & 15.8965742135 \\
\hline 10 & 11.7264573154 \\
\hline Average & 14.39903242044 \\
\hline
\end{tabular}

By performing the above operations concurrently with the multiprocessing command, the following result is appeared on the screen:
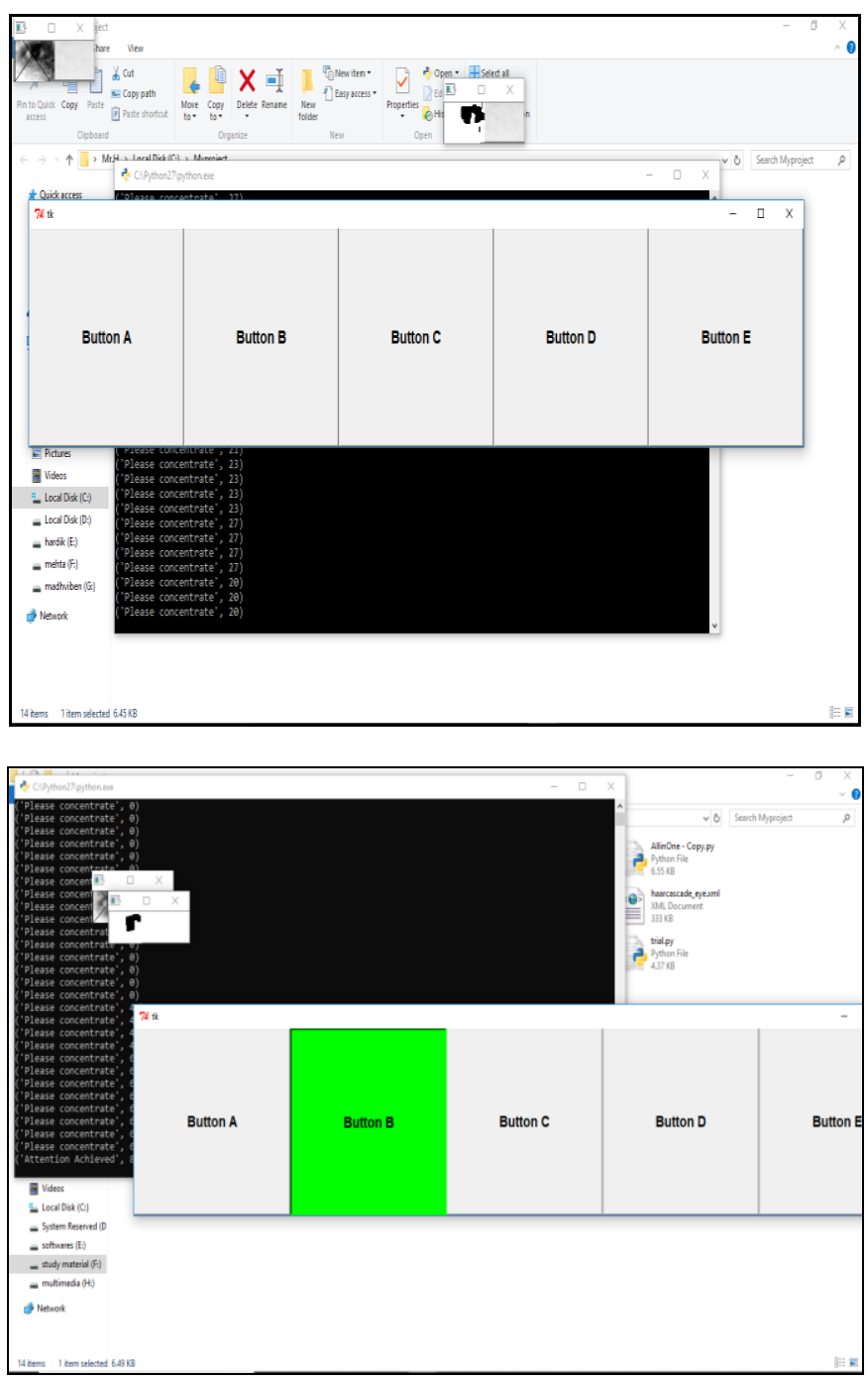

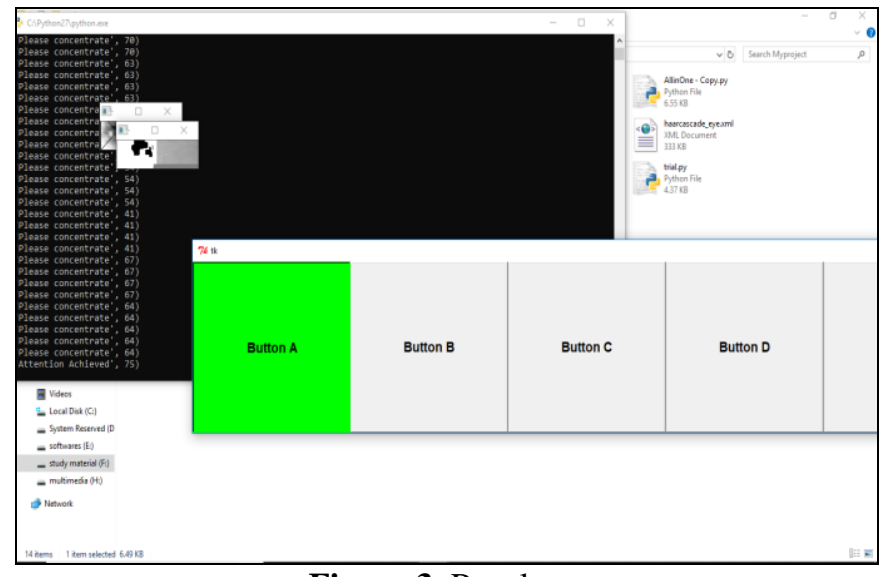

Figure 3. Results

In the Fig.3, it is shown that there are two windows of Eye pupil detection: one is in the original form and another is in the gray scale form. To detect the Eye pupil properly, it is necessary to convert the original frame into gray scale form. Moreover, attention values are shown in the terminal window. Till the attention value reaches to 70 , it is written that 'Please Concentrate'. Once it crosses 70 and goes above, it is written that 'Attention is archived' and mouse is clicked where the mouse pointer is situated on the GUI (5 buttons) shown in the figure and when a click is performed, GUI button changes into green colored button.

Benefits of using BCI is that when user wants to click, then he has to concentrate on that point for a while so that Attention Values go beyond the threshold level and it clicks where the mouse cursor is situated. Another approach to perform the mouse clicks without BCI is that when a user performs 3 or 4 blinks within 1 second, then algorithm should click the mouse automatically. But the disadvantage with this method is that this work is specially proposed to the patients with physical disabilities. So if the patient blinks too much times occasionally or when sometimes any insect comes to near his eyes like bees, mosquitoes then he will also have to blink in order to protect his eyes. So all these kinds of situations perform unnecessary clicks. But by using BCI, all these limitations can be removed successfully.

\section{Conclusion and Future Scope}

Physically disabled people suffer from depression. Use of MindWave headset has been proved to be very useful for physically disabled people to communicate with the world. Attachment of Eye Gaze Tracker with BCI has increased its functionality. In addition to that, a GUI increases comfortability to the patients for doing the predefined tasks.

In future, there is a need to build such an algorithm which can stabilize the position of a mouse cursor for operating a whole computer because this can be achieved by the head mounted eye gaze tracker but it makes the patients feel uncomfortable. Furthermore this project can be expanded by 
making an application specific device for it. This can also be used in robotic arm, automatic wheelchair, video games etc.

\section{References}

1. Hassanien, Aboul Ella, and A. A. Azar. "BrainComputer Interfaces." Switzerland: Springer (2015).

2. Rabie A. Ramadan, S. Refat, Marwa A. Elshahed and Rasha A. Ali, "Basics of Brain Computer Interface", Intelligent Systems Reference Library 74, DOI 10.1007/978-3-319-10978-7_2

3. Stephygraph, L. Ramya, N. Arunkumar and V. Venkatraman. "Wireless mobile robot control through human machine interface using brain signals." Smart Technologies and management for Computing, Communication, Controls, Energyand Materials (ICSTM), 2015 International Conference on. IEEE, 2015

4. Jones, Ashley, and Galina Schwartz. "Using braincomputer interfaces to analyze EEG data for safety improvement." Team for Research in Ubiquitous Secure Technology (2010).

5. Devashish Salvekar, Amrita Nair, Dany Bright, Prof. S. A. Bhisikar, "Mind Controlled Robotic Arm", IOSR Journal of Electronics and Communication Engineering (IOSR-JECE) e-ISSN: 2278-2834,pISSN: 2278-8735. PP 36-44 www.iosrjournals.org
6. Keerthana, Aravind, Karmel, "Controlling Electrical Devices with Human Brainwaves", VIT University Chennai, INDIA.

7. Kumar, Ankit, Joy Bose, and Divya Bansal. "A web browser responsive to the user interest level." India Conference (INDICON), 2015 Annual IEEE. IEEE, 2015.

8. Gelšvartas, J., Simutis, R., \& Maskeliūnas, R. (2015, August). Multifunctional user interface implementation details and evaluation. In Methods and Models in Automation and Robotics (MMAR), 2015 20th International Conference on (pp. 501504). IEEE.

9. Castillo, Aaron, Graciela Cortez, David Diaz, Rayton Espíritu, Krystle Ilisastigui, Bryce O'Bard, and Kiran George. "Hands free mouse." In Wearable and Implantable Body Sensor Networks (BSN), 2016 IEEE 13th International Conference on, pp. 109114. IEEE, 2016.

10. Viola, Paul, and Michael Jones. "Rapid object detection using a boosted cascade of simple features." Computer Vision and Pattern Recognition, 2001. CVPR 2001. Proceedings of the 2001 IEEE Computer Society Conference on. Vol. 1. IEEE, 2001

11. Timm, Fabian, and Erhardt Barth. "Accurate Eye Centre Localisation by Means of Gradients." Visapp 11 (2011): 125-130

12. www.neurosky.com/MindWave_mobile_user_guide.pdf

\section{Biographical notes}

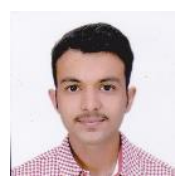

H. M. Mehta is pursuing M.E. in Communication System Engineering from B. H. Gardi College of Engineering and Technology, Rajkot, Gujarat, India. His research interest includes Embedded Systems, Digital Image Processing and Video Processing.

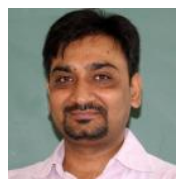

Prof. M. P. Patel is the Head of the Department in B. H. Gardi College of Engineering and Technology, Rajkot, Gujarat, India. His research interest includes Digital Image Processing, Digital Signal Processing, Information Theory and Coding.

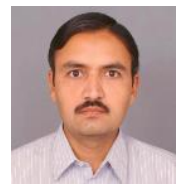

Dr. C. H. Vithalani is the Head of the Department in Government Engineering College, Rajkot, Gujarat, India. His research interest includes Digital Image Processing and Embedded systems. 